THE exact causes of the extensive cell death in nigral transplants are still unknown. Since poly-(ADP-ribose) polymerase (PARP) overactivation has been implicated in neuronal death, we examined the effects of PARP on the survival of nigral grafts by using donor tissue from PARP knock-out or wild-type mice. Eight hours after preparation of the nigral cell suspension, cell damage was quantified by measurement of lactate dehydrogenase release, DNA fragmentation and caspase activation. At this stage, PARP deletion had no protective effect. Moreover, neither the survival of transplanted dopaminergic neurons, nor the functional recovery of hemiparkinsonian graft recipients were improved by the absence of PARP. We conclude that cell death in embryonic nigral grafts is not affected by the absence of PARP activation.

Key words: Apoptosis; Dopamine; Necrosis; Neural transplantation; Parkinson's disease; PARP; Substantia nigra

\section{Neuronal death in nigral grafts in the absence of poly (ADP-ribose) polymerase activation}

\author{
Gabriele S. Kaminski Schierle, ${ }^{\mathrm{CA}}$ \\ Oskar Hansson, Elisa Ferrando-May, ${ }^{1}$ \\ Pierluigi Nicotera, ${ }^{1}$ Patrik Brundin and \\ Marcel Leist ${ }^{1}$
}

\author{
Section for Neuronal Survival, Wallenberg \\ Neuroscience Center, Department of Physiological \\ Sciences, Lund University, Sölvegatan 17, SE-223 \\ 62 Lund, Sweden; ${ }^{1}$ Department of Molecular \\ Toxicology, Faculty of Biology, Konstanz \\ University, Box X911, D-78457 Konstanz, \\ Germany
}

${ }^{\mathrm{CA}}$ Corresponding Author

\section{Introduction}

Implantation of dopaminergic neurons from the human embryonic ventral mesencephalon (VM) into the denervated neostriatum of Parkinson's disease (PD) patients has developed into a powerful approach to treat this devastating illness $[1,2]$. The survival of transplanted dopaminergic neurons is typically about $5-15 \%$ in experimental animals and appears to be even less in humans (reviewed in [3]). Because the availability of suitable donor tissue is a major limiting factor for clinical intracerebral transplantation, there is a need to improve the survival of transplanted dopaminergic neurons.

Recent studies have suggested that most neurons die during and shortly after transplantation [4-6], and that up to $40 \%$ of dopaminergic neuron death may be inhibited when apoptosis is blocked by caspase inhibition [5]. However, the mode and mechanisms of death responsible for the loss of most of the cells remain unknown.

Poly-(ADP-ribose) polymerase (PARP; EC 2.4.4.30) is a nuclear enzyme involved in the maintenance of chromatin integrity [7]. During apoptosis PARP is frequently cleaved and inactivated at early stages [8]. Deletion of PARP has no effect on the extent of apoptotic cell death elicited by a variety of different stimuli $[9,10]$. In contrast, overactivation of PARP, e.g. by DNA strand breaks, may lead to cellular energy deficit followed by non-apoptotic cell death $[11,12]$. The assumption that PARP may be relevant for some forms of necrotic cell death is supported by the findings that mice with a deleted PARP gene (PARP-/-) $[13,14]$ are protected from neuronal necrosis following transient cerebral ischemia $[15,16]$ and $\mathrm{MPP}^{+}$intoxication [17]. Furthermore, neurons from PARP-/- mice display resistance to peroxynitrite, glutamate, and other compounds that normally trigger necrosis (reviewed in $[11,12])$.

We hypothesized that PARP activation may be responsible for the caspase-independent part of cell death in nigral transplants, and that its inhibition may improve the efficacy of neuronal grafting. To test the usefulness of this potential therapeutic intervention, we examined the immediate cell death in embryonic mesencephalic cell suspensions from wild-type (wt) and PARP-/- mice prior to intracerebral transplantation. Then, we evaluated whether PARP deletion improves the survival of transplanted dopaminergic neurons as well as the functional efficacy of the grafts. 


\section{Materials and Methods}

Animals: PARP $-/-$ mice were initially generated [14] and kindly provided by Dr Z.-Q. Wang (IARC, Lyon, France). These animals were cross-bred with $\mathrm{C} 57 \mathrm{BL} / 6(\mathrm{wt})$ mice and the resulting heterozygous litters (F1) were used for further inbred breeding. Offspring from heterozygous matings (F2) were genotyped by PCR. For preparation of transplant suspensions, wild-type (wt) or homozygous PARP-/- F2 animals were used and embryonic tissue was obtained 13 days (E13) after mating. All steps of animal handling were performed according to the Guidelines of the EC (European council directive 86/609/EEC) and followed the directives of the Lund University ethical committee.

Ventral mesencephalic cell suspensions: Ventral mesencephalic donor tissue from E13 PARP-/- and wt mice was dissected and dissociated as described for rats [18]. Cell suspensions were either further processed for transplantation or diluted to $1 \times 10^{5}$ cells/ml HBSS (Gibco, Life Technologies, Taeby, Sweden) for in vitro experiments. To evaluate the initial percentage of tyrosine-hydroxylase (TH)immunopositive neurons in the cell suspensions, cell smears $(n=3$ in each) were prepared on gelatincoated slides, dried and fixed with formalin. They were processed for TH staining, and assessed in the light microscope as described previously [18].

Viability parameters and enzymatic assays: Ventral mesencephalic suspensions were kept for $6 \mathrm{~h}$ at room temperature followed by $2 \mathrm{~h}$ at $37^{\circ} \mathrm{C}$. The initial cell number and viability ( $>98 \%$ viable cells) as well as the number of remaining cells after $8 \mathrm{~h}$ were assessed using trypan blue dye exclusion. In addition, cell suspensions were processed for measurement of DNA fragmentation ( $n=3$ in each) and lactate dehydrogenase $(\mathrm{LDH})$ release $(n=3$ in each) as described previously $[5,19]$. The $100 \%$ reference value for DNA fragmentation was derived from analysis of ventral mesencephalic suspensions immediately after preparation $(t=0)$. The percentage of $\mathrm{LDH}$ released from the cells is indicated with reference to the total $\mathrm{LDH}$ activity initially present in the cell suspensions. Caspase activity $(n=5$ in each) was determined fluorimetrically, using DEVD-7-amino-3-trifluoromethylcoumarin as substrate (DEVD-afc) [20].

Immunoblotting: For immunoblot analysis, freshly dissected tissue or ventral mesencephalic cell suspensions were snap-frozen in liquid nitrogen. They were homogenized in $100 \mu$ l RIPA buffer $(150 \mathrm{mM}$ $\mathrm{NaCl}, 50 \mathrm{mM}$ Tris, $1 \%$ NP-40, $0.25 \%$ sodium deoxycholate, $1 \mathrm{mM}$ EGTA) supplemented with inhibitors of proteases ( $1 \mathrm{mM}$ PMSF, $1 \mathrm{mM}$ benzamidine, $1 \mathrm{mM}$ iodoacetate, $1 \mathrm{mM}$ iodoacetamide, $40 \mu \mathrm{M}$ leupeptin, $10 \mu \mathrm{g} / \mathrm{ml}$ antipain, $5 \mu \mathrm{g} / \mathrm{ml}$ pepstatin). Protein content of the homogenates was determined by the bicinchoninic acid method (Biorad, Munich, Germany). Equal amounts of protein were separated by SDS-polyacrylamide gel electrophoresis, and electroblotted onto nitrocellulose membranes ( $\mathrm{Hy}$ bond ECL, Amersham-Buchler Corp., Braunschweig, Germany). Protein content was routinely controlled by staining of blot membranes with Ponceau Red and by redeveloping the membranes with an anti-actin antibody (1:100000; Chemicon Inc., Temecula, CA, clone C4). The membranes were blocked and incubated with the primary antibodies (PARP monoclonal (murine) clone C2-10, Pharmingen). Blots were developed with IgGhorseradish peroxidase followed by enhanced chemiluminescence detection (ECL, Amersham).

Unilateral 6-bydroxydopamine lesion and motor asymmetry test: Female Sprague-Dawley rats (B\&K Universal AB, Sollentuna, Sweden) were subjected to unilateral 6-hydroxydopamine (6-OHDA; Sigma, Sweden) lesions of the ascending mesostriatal pathway [18]. Rats exhibiting a net rotational amphetamine $(2.5 \mathrm{mg} / \mathrm{kg}$ D-amphetamine)-induced asymmetry of at least six full turns/min were selected for transplantation surgery. Rats were tested for amphetamine-induced rotations 2 and 5 weeks after grafting. Data for each rat at the two posttransplantation tests were expressed as a percentage of the pre-transplantation score.

Transplantation surgery: Two groups of rats with 6-OHDA lesions ( $n=6$ in each) were transplanted with ventral mesencephalic cell suspensions prepared from either PARP $-/-$ or wt donor embryos. Two deposits of $1.5 \mu \mathrm{l}$ cell suspension $(\sim 125000$ cells in total) were injected stereotaxically under equithesin anesthesia into the right (lesioned) striatum of rats, at the following co-ordinates (according to bregma): A: $+1 \mathrm{~mm}, \mathrm{~L}:-3 \mathrm{~mm}, \mathrm{~V}:-5 \mathrm{~mm}$ and $-4.5 \mathrm{~mm}$, with the tooth-bar set at zero. Following transplantation, the rats received daily intraperitoneal injections of $10 \mathrm{mg} / \mathrm{kg}$ cyclosporin A (Sandimmun, Novartis, Taeby, Sweden) to reduce the risk of xenograft rejection [21].

Histological assessment: Five weeks after transplantation surgery, rats were anaesthetized, perfused, and the brains were processed for $\mathrm{TH}$-immunocytochemistry [18]. The number of TH-positive neurons in the grafts was counted in serial sections on blindcoded slides using a semi-automated stereological 
cell counting system [5]. The total number of surviving TH-positive neurons in the graft and the total graft volume were extrapolated according to previously used stereological algorithms [22].

\section{Results}

Expression of PARP and proportion of TH-immunopositive neurons in embryonic tissue: The VM and other brain regions of E13 mice were examined by immunoblot for the presence of PARP protein. PARP was detected in abundant quantities in wt brain tissue (Fig. 1A) which indicates that the parp gene was already transcribed at this developmental stage. Notably, the absence of the protein did not significantly affect the percentage of $\mathrm{TH}$-positive neurons in VM cell suspensions $(8.7 \pm 0.3 \% \mathrm{TH}$ positive in PARP-/ - vs $9.1 \pm 0.6 \% \mathrm{TH}$-positive in wt mice; $n=3$ in each). PARP was also abundantly present in wt ventral mesencephalic suspensions and remained stable there during an incubation period of up to $8 \mathrm{~h}$ (Fig. 1B).

Cell death in ventral mesencephalic cell suspensions in the absence of PARP: To model pre-and peritransplantational stress, ventral mesencephalic suspensions from either PARP-/- or wt mice were left at room temperature for $6 \mathrm{~h}$ and then incubated for $2 \mathrm{~h}$ at $37^{\circ} \mathrm{C}$. This treatment led to a similar increase in the activity of DEVD-afc cleaving caspases in cells of both genotypes (two-factor ANOVA, effect of genotype, $p>0.05, \mathrm{~F}(1,16)=$ $0.12 ; n=5$ in each; Fig. 2B). The amount of oligo-

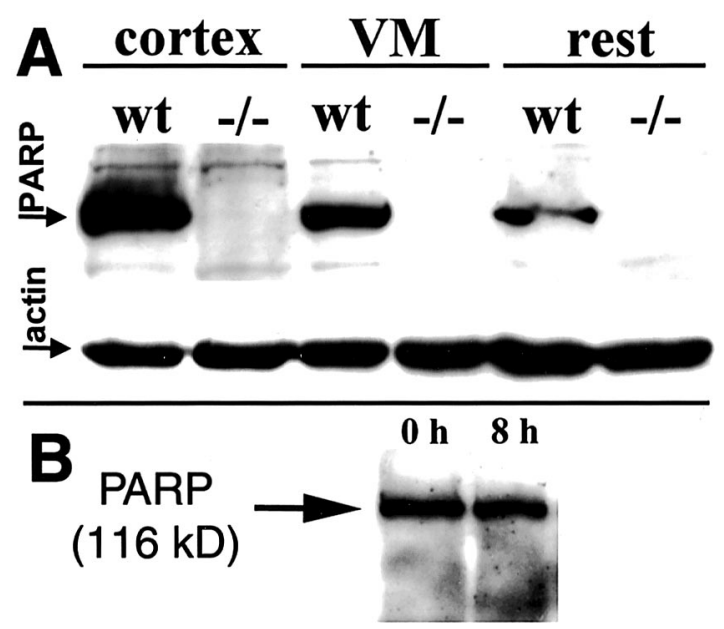

FIG. 1. Expression of PARP in embryonic mesencephalic tissue. (A) Embryonic brains (E13) from wt and PARP-/- animals were dissected into cortex, VM and the rest of the brain (rest) and analyzed for PARP by Western blotting. Actin $(42 \mathrm{kDa})$ was used as a loading control. (B) Ventral mesencephalic cell suspensions that had been incubated for 0 or $8 \mathrm{~h}$ were centrifuged, homogenized and analyzed for the content of intact PARP protein. PARP was not significantly degraded during the $8 \mathrm{~h}$ incubation.
A

B

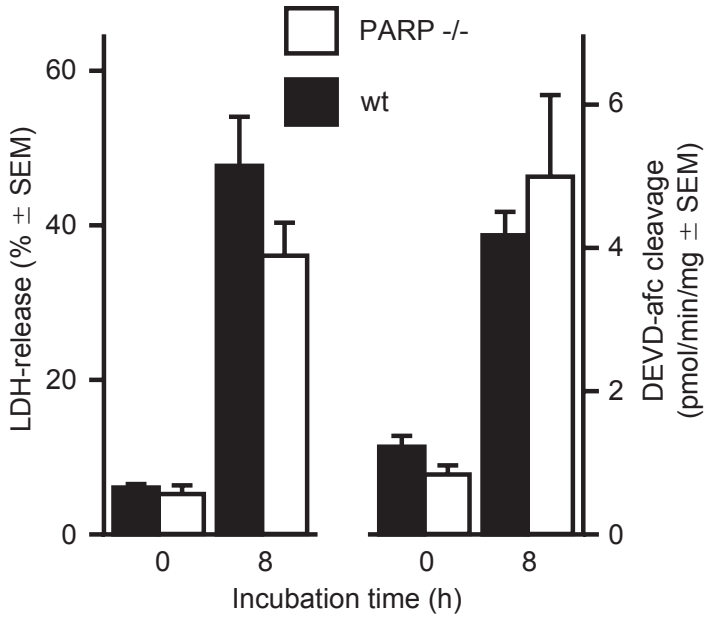

FIG. 2. No prevention of cell death in ventral mesencephalic cell suspensions in the absence of PARP. PARP-/- or wt ventral mesencephalic cell suspensions were either analyzed immediately $(0 \mathrm{~h})$ or stored for $6 \mathrm{~h}$ at room temperature followed by $2 \mathrm{~h}$ at $37^{\circ} \mathrm{C}(8 \mathrm{~h})$ before $(\mathbf{A}) \mathrm{LDH}$ release $(n=3$ in each) or (B) DEVD afc cleavage $(n=5$ in each) was determined.

nucleosomal DNA fragments in cell suspensions from wt $(n=3 ; 6070 \pm 700 \%$, s.e.m. $)$ or PARP knock-out $(n=3 ; 5870 \pm 450 \%)$ animals (freshly prepared control cells $=100 \%$ ) was also similar after $8 \mathrm{~h}$. When we investigated the overall cell lysis within the experimental period $(8 \mathrm{~h})$ by measuring the amount of $\mathrm{LDH}$ released into the medium from dying cells, no significant change in the survival of PARP - / - cells was observed (two-factor ANOVA, effect of genotype, $p>0.05, \mathrm{~F}(1,8)=2.41 ; n=3$ in each; Fig. 2A).

Effects of PARP deletion on the survival and function of dopaminergic neurons in nigral grafts: We further examined whether dopamine-rich transplants of PARP-/ - ventral mesencephalic tissue would survive better than those derived from wt tissue, and whether functional recovery in a rat model of PD would be enhanced by PARP deletion. Our data showed that rats receiving PARP-/- or wt grafts recovered at a similar rate and to the same extent from their lesion-induced asymmetry (Fig. 3A; two-factor ANOVA, effect of genotype, $p>0.05$, $\mathrm{F}(1,10)=0.94 ; n=6$ in each).

Histological examination of the grafted brains revealed that striata from rats in both the wt and the PARP $-/$ - groups contained surviving graft tissue with numerous $\mathrm{TH}$-immunopositive neurons that extended fibers into the surrounding denervated host striatum (Fig. 3B,C). Cell counts revealed no difference in the number of surviving dopaminergic neurons in the PARP $-/-$ grafts $(n=6 ; 1740 \pm 240)$ compared with wt grafts $(n=6 ; 1730 \pm 390)$. Thus, 
A
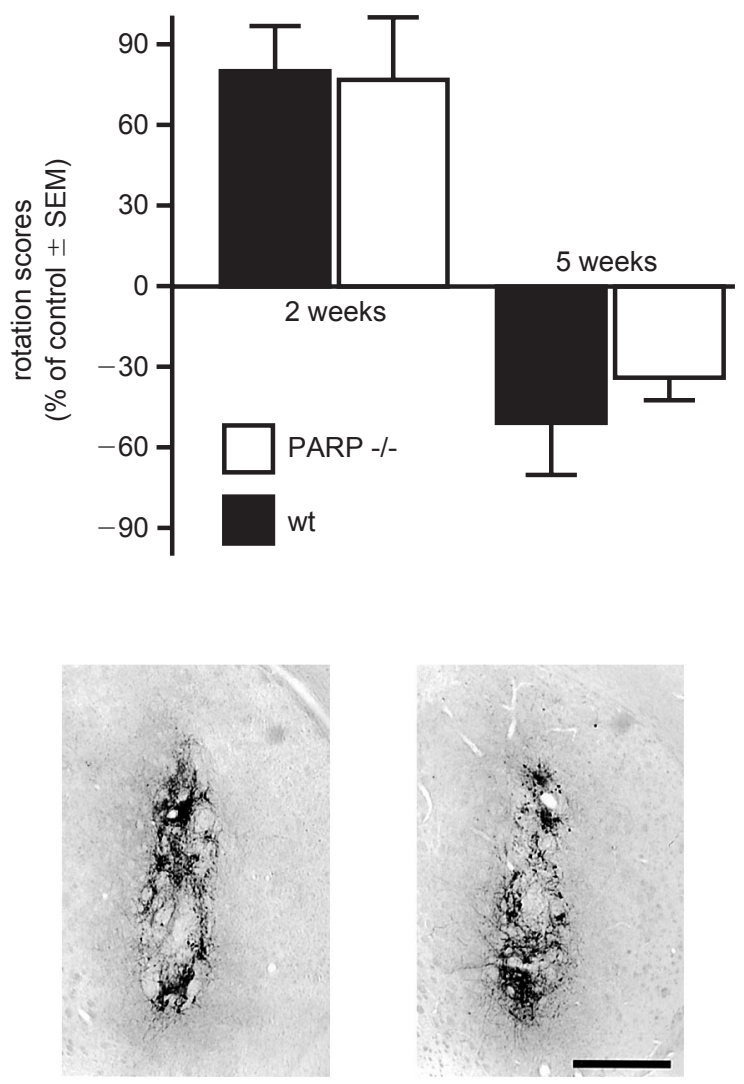

FIG. 3. Effects of PARP deletion on the survival and function of dopaminergic neurons in mesencephalic grafts. (A) Net ipsilateral rotation scores of hemiparkinsonian rats were analyzed 2 and 5 weeks after grafting with wt or PARP $-1-$ tissue ( $n=6$ each). Both groups exhibited a similar reversal of motor asymmetry over time. Data are expressed as percentage of pretransplantation scores $(=100 \%$ control value). (B,C) Photomicrographs showing tyrosine hydroxylase $(\mathrm{TH})$ immunostaining of representative coronal sections through the striatum of the host brain receiving wt or PARP-/- grafts, respectively. Bar $=0.56 \mathrm{~mm}$.

the survival rate of about $15 \pm 2 \%$ of $\mathrm{TH}$-positive neurons was not altered by PARP deletion.

\section{Discussion}

An increased understanding of the mechanisms leading to dopaminergic neuron death in grafts would facilitate the search for new neuroprotective strategies [3]. We have recently shown that apopotosis is initiated within hours after tissue preparation for nigral transplantation [5], and although it involves caspase activation [5] it does not involve a Bcl-2sensitive step [19]. We have further shown that the survival of grafted neurons is significantly increased by treatment with a caspase inhibitor. However, counts of viable dopaminergic neurons in such grafts indicated that $\geqslant 60 \%$ of the initially prepared neurons are still lost [5]. Our present experiments show that PARP activation cannot account for the loss of grafted nigral neurons, i.e. the absence of PARP neither reduced the early cell death in transplant suspensions nor affected the long term survival and functionality of grafts compared to wt grafts. Moreover, there did not seem to be a significant effect on the way in which cells died [23] in the presence or absence of PARP, since formation of oligonucleosomal fragments or caspase activation were unaffected.

In recent years, PARP has attained a great deal of attention as a potential target for neuroprotective strategies (reviewed in [11,12]), and some involvement of PARP activation in the massive cell death following neuronal grafting appeared likely. However, it has become clear that PARP inhibition selectively rescues specific types of cells exposed to a particular type of stress. For instance, apoptotic death is typically little affected by PARP deletion $[9,10,16,20]$, whereas nitric oxide-mediated neuronal necrosis and various other types of ischemia-related cell death appear to be mediated by PARP activation $[11,12]$. The lack of any protection in PARP-/nigral grafts suggests that here cell death seems to involve different mechanisms. In fact, formation of NO has been shown to be irrelevant to the loss of grafted neurons [24].

\section{Conclusion}

Our in vivo and in vitro experiments suggest that PARP activation does not contribute to neuronal cell death in nigral transplants. Thus, inhibition of PARP is not a universal neuroprotective strategy, in particular when apoptosis appears to account for a large part of the neuronal loss.

\section{References}

1. Lindvall O. Neuroreport 8, iii-x (1997).

2. Tabbal S, Fahn S and Frucht S.Curr Opin Neurol 11, 341-349 (1998).

3. Brundin $P$ and Kaminski Schierle GS. Neuroprotective strategies in neural grafting. In: Dunnett SB, Boulton AA and Baker GB, eds. Neural Transplantation Methods. Totowa, NJ: Humana Press Inc, 1999. 411-440.

4. Widner $\mathrm{H}$ and Brundin P. Exp Brain Res 104, 227-242 (1995).

5. Schierle GS, Hansson O, Leist M et al. Nature Med 5, 97-100 (1999).

6. Zawada WM, Zastrow DJ, Clarkson ED et al. Brain Res 786, 96-103 (1998)

7. de Murcia G and Ménissier de Murcia J. Trends Biochem Sci 19, 172-176 (1994)

8. Lazebnik YA, Kaufmann SH, Desnoyers S et al. Nature 371, 346-347 (1994).

9. Leist M, Single B, Kunstle G et al. Biochem Biophys Res Commun 334, 518-522 (1997).

10. Wang Z-Q, Stingl L, Morrison C et al. Genes Dev 11 2347-2358 (1997).

11. Szabo C and Dawson VL. Trends Pharmacol Sci 19, 287-298 (1998).

12. Pieper AA, Verma A, Zhang J and Snyder SH. Trends Pharmacol Sci 20, 171-181 (1999).

13. de Murcia JM, Niedergang C, Trucco C et al. Proc Natl Acad Sci USA 94 7303-7307 (1997).

14. Wang Z-Q, Auer B, Stingl L et al. Genes Dev 9, 509-520 (1995).

15. Eliasson MJL, Sampei K, Mandir AS et al. Nature Med 3, 1089-1095 (1997).

16. Endres M, Wang ZQ, Namura $S$ et al. J Cerebr Blood Flow Metab 17, 1143-1153 (1997).

17. Mandir AS, Przedborski S, Jackson-Lewis V et al. Proc Natl Acad Sci USA 96, 5774-5779 (1999).

18. Karlsson J, Emgard M, Rosenblad C, Brundin P. Brain Res 805, 155-168 (1998).

19. Schierle GS, Leist M, Martinou JC et al. Eur J Neurosci 11, 3073-3081 (1999).

20. Leist M, Volbracht C, Kuhnle S et al. Mol Med 3, 750-764 (1997).

21. Brundin P, Nilsson OG, Gage FH and Bjorklund A. Exp Brain Res 60, 204-208 (1985).

22. West MJ, Slomianka L and Gundersen HJ. Anat Rec 231, 482-497 (1991). 
23. Nicotera P, Leist M and Manzo L. Trends Pharmacol Sci 20, 46-51 (1999).

24. Van Muiswinkel FL, Drukarch B, Steinbusch MW and De Vente J. Brain Res 792 48-58 (1998)

ACKNOWLEDGEMENTS: We gratefully acknowledge the technical assistance of Birgit Haraldsson, Heike Naumann, Britt Lindberg and Bengt Mattsson. This study was supported by grants from the Swedish Medical Research Council, and DFG and EEC grants to P.N. and M.L. G.S.K.S. was supported by a grant of the Parkinson's
Disease Foundation and O.H. was supported by the National Network in Neuroscience. 